\title{
Supporting Information Interactions of microorganisms with lipid Langmuir layers
}

Avia Leader ${ }^{[1]}$, Ori Molad ${ }^{[1,2]}$, Aviv Dombrovsky ${ }^{[2]}$, Meital Reches ${ }^{[1] *}$ and Daniel Mandler ${ }^{[1] *}$

[1] The Institute of Chemistry and Center for Nanoscience and Nanotechnology

The Hebrew University of Jerusalem, 91904 Jerusalem, Israel

Edmond Safra campus, Jerusalem 9190401, Israel

E-mail: daniel.mandler@mail.huji.ac.il, meital.reches@mail.huji.ac.il

[2] Department of Plant Pathology and Weed Research, Agricultural Research Organization, The Volcani Center, 68 HaMaccabim Road, P.O.B 15159 Rishon LeZion 7505101

Correspondence: e-mail: aviv@volcani.agri.gov.il; Tel. : 972-3-9683579; Fax: 972-3-9686543.
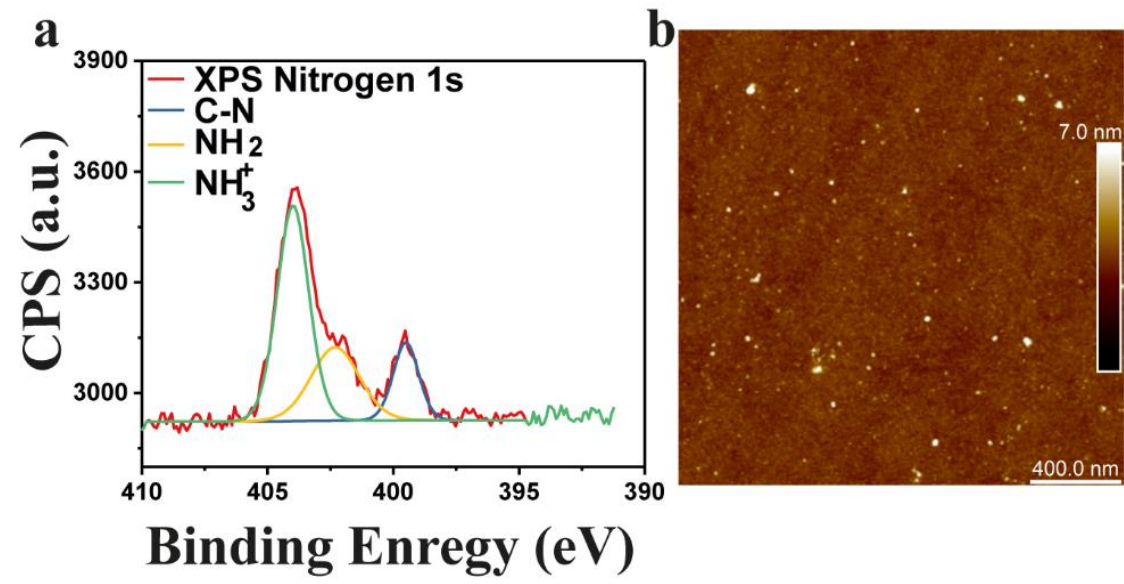

Figure S1. XPS and AFM analysis of APTES films on a silicon substrate. The N 1s spectrum of the coated surface with common peaks at 400, 402 and $404 \mathrm{eV}$, which are attributed to $\mathrm{C}-\mathrm{N}, \mathrm{NH}_{2}$ and $\mathrm{NH}_{3}{ }^{+}$groups, respectively (a). AFM topography image of the films with the roughness of $0.174 \mathrm{~nm}(\mathrm{~b})$.

Zeta Potential Distribution

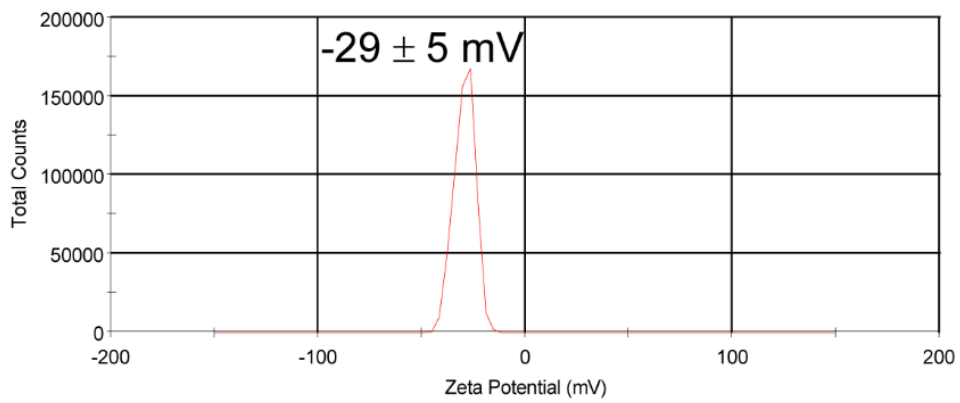

Figure S2. Zeta potential distribution of CGMMV in a PBS solution. 


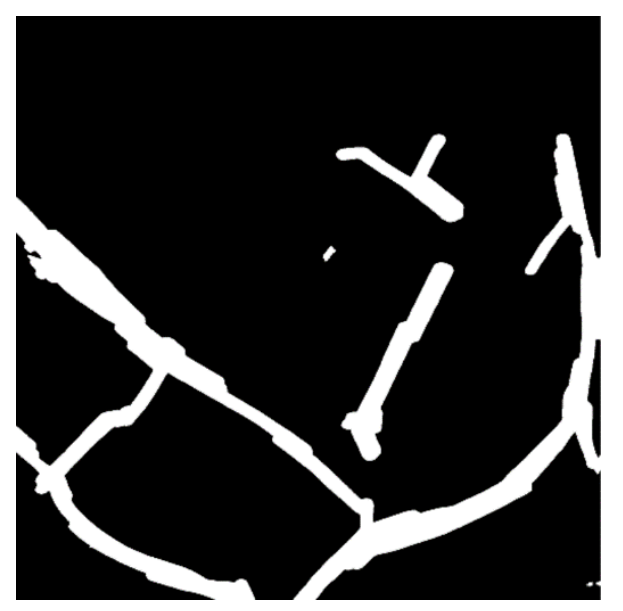

Figure S3. A representative ImageJ analysis of an AFM image of a deposited LB layer of DODAB and CGMMV virions.

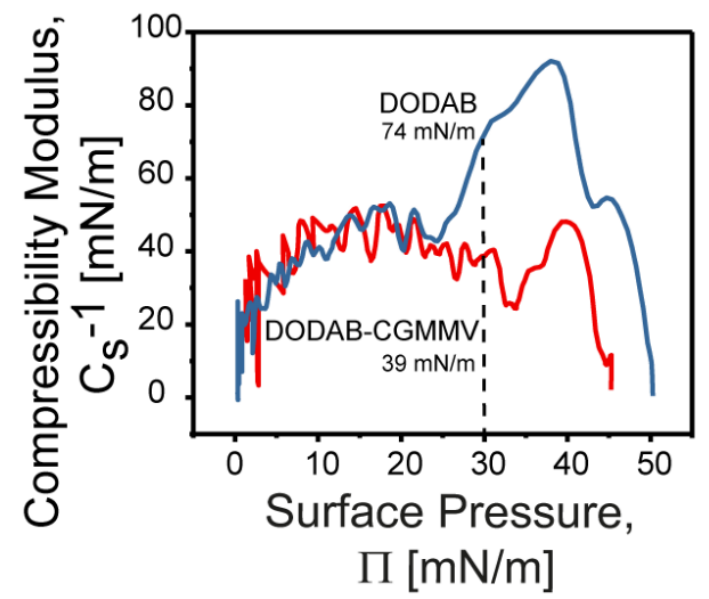

Figure S4. Compressibility modulus of pure DODAB and DODAB incorporated with CGMMV virions. 